\title{
Microsurgical robotic suturing of sural nerve graft for sympathetic nerve reconstruction: a technical feasibility study
}

\author{
Tommy Nai-Jen Chang ${ }^{1}$, Lisa Wen-Yu Chen ${ }^{1}$, Chin-Pang Lee ${ }^{2}$, Kuo-Hsuan Chang ${ }^{3}$, \\ David Chwei-Chin Chuang ${ }^{1}$, Yin-Kai Chao ${ }^{4}$
}

${ }^{1}$ Department of Plastic and Reconstructive Surgery, Chang Gung Memorial Hospital, Chang Gung Medical College and Chang Gung University, Taoyuan; ${ }^{2}$ Department of Psychiatry, ${ }^{3}$ Department of Neurology, ${ }^{4}$ Division of Thoracic Surgery, Chang Gung Memorial Hospital-Linko, Chang Gung University, Taoyuan

Contributions: (I) Conception and design: T Chang, YK Chao; (II) Administrative support: YK Chao; (III) Provision of study materials or patients: T Chang, YK Chao; (IV) Collection and assembly of data: L Chen, CP Lee; (V) Data analysis and interpretation: T Chang, YK Chao; (VI) Manuscript writing: All authors; (VII) Final approval of manuscript: All authors.

Correspondence to: Yin-Kai Chao, MD. Division of Thoracic Surgery, Chang Gung Memorial Hospital-Linko, Chang Gung University, Taoyuan. Email: chaoyk@cgmh.org.tw.

\begin{abstract}
Background: Endoscopic thoracic sympathectomy (ETS) may provide a permanent surgical cure for primary palmar hyperhidrosis. Unfortunately, some patients can experience intensive post-operative compensatory sweating (CS) that ultimately impairs quality of life. Sympathetic nerve reconstruction (SNR) may be used to counteract severe post-operative CS through the restoration of sympathetic pathways. In this case series, we describe the technical feasibility of a robot-assisted micro-peripheral nerve reconstruction method for achieving SNR in patients with post-operative CS.

Methods: Between January 2017 and May 2019, seven cases with severe post-operative CS underwent robot-assisted SNR using a sural nerve graft. We report the pre-operative assessment, the surgical technique, and the clinical outcomes of the study patients.

Results: The study sample consisted of five men and two women (median age: 41 years). Primary hyperhidrosis affected the face in one case and the palms in six patients. The median time between ETS and SNR was 20 years. All robotic surgery procedures were successfully accomplished, and neither conversion to open surgery nor the creation of additional ports were required. Sural nerve grafts (median length: $8 \mathrm{~cm}$ ) were used in all cases, and the median operating time was $10.5 \mathrm{~h}$. There was no operative mortality, with the median length of post-operative hospital stay being 4 days. One patient developed a post-operative pneumothorax-which was treated conservatively.

Conclusions: Our case series demonstrates the safety and clinical feasibility of microsurgical robot-assisted sural nerve grafting for achieving SNR in patients with post-operative CS.
\end{abstract}

Keywords: Robotic microsurgery; sympathetic nerve reconstruction (SNR); endoscopic thoracic sympathectomy (ETS); compensatory sweating (CS)

Submitted Jul 09, 2019. Accepted for publication Aug 02, 2019.

doi: $10.21037 /$ jtd.2019.08.52

View this article at: http://dx.doi.org/10.21037/jtd.2019.08.52

\section{Introduction}

Endoscopic thoracic sympathectomy (ETS) may provide a permanent surgical cure for primary palmar hyperhidrosis. Unfortunately, some patients can experience intensive post- operative compensatory sweating (CS) - a sympathetic trunk disruption-related manifestation that ultimately impairs quality of life (1). Although sympathetic nerve reconstruction (SNR) allows counteracting severe postoperative CS through the restoration of sympathetic 


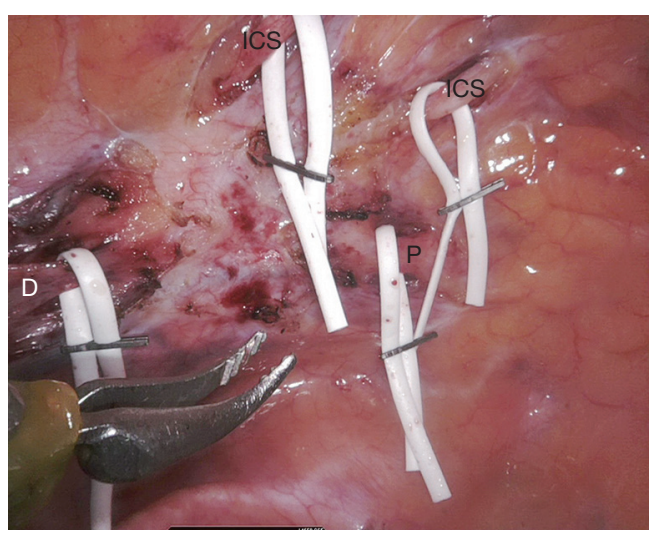

Figure 1 Representative images obtained before sympathetic nerve reconstruction. The scarred proximal (P) and distal (D) ends of the sympathetic trunk and the affected second-to-fourth intercostal (ICS) nerves were identified using white vessel loops.

pathways (2), no definite conclusions can currently be drawn as to its clinical utility $(3,4)$.

Current surgical robots are not only capable of providing three-dimensional stereoscopic vision (10x magnification) but can also perform wrist-like movements-ultimately allowing fine dissections and suturing even in confined anatomical spaces.

In the present case series, we describe the technical feasibility of a robot-assisted nerve reconstruction method for achieving SNR in patients with post-operative CS. We specifically focused on the pre-operative assessment, surgical technique, and clinical outcomes.

\section{Methods}

\section{Study patients}

We conducted a retrospective chart review of patients who underwent robotic SNR at the Department of Thoracic Surgery, Chang Gung Memorial Hospital (Linko) between January 2017 and May 2019. All operations were performed by a single surgical team consisting of both thoracic surgeons and microsurgeons.

\section{Pre-operative planning}

A thorough medical history-with a special focus on (I) clinical symptoms before and after ETS and (II) the technique used to interrupt the sympathetic chain-was collected in all cases. Physical examination was aimed at determining (I) the presence or absence of the Horner syndrome, (II) the anatomical distribution of sweating, and (III) the relative temperature of the extremities. Patients with exercise limitations underwent an exercise-limited cardiac stress test. All candidates for SNR underwent an extensive presurgical psychological assessment by an experienced psychiatrist.

We did not recommend SNR to patients without a history of non-surgical sympathectomy (e.g., achieved through thermal ablation or chemical injections) because it can be difficult to precisely determine the extent of the original thermal or chemical damage, ultimately questioning the clinical utility of reversal surgery.

After a detailed explanation of potential risks inherent to SNR-including the reappearance of symptoms that originally prompted ETS, the occurrence of potential injuries to the sympathetic chain-all participants gave their written informed consent. Several patients who requested to undergo SNR had an interruption at the level of the second rib—requiring the "freshening up" of the proximal nerve immediately below the stellate ganglion at the time of nerve reconstruction. We informed patients about the risks of direct or traction injuries to the stellate ganglion, resulting either in a transient or permanent component of the Horner syndrome.

\section{Surgical technique}

Upon induction of general anesthesia and intubation with a double-lumen endotracheal tube, the patient was placed in the semi-prone position. Upon creation of suitable thoracic ports, the following trocar positions were used: (I) a 30-degree angled thoracoscope was positioned in the seventh intercostal space (ICS) along the posterior axillary line; (II) the right robotic arm was placed in the fifth ICS along the posterior axillary line, medially to the scapula; (III) the left robotic arm was positioned in the ninth ICS along the posterior axillary line; and (IV) the assistant port was placed either in the sixth or seventh ICS along the mid axillary line. A da Vinci ${ }^{\circledR}$ Xi robotic cart (Intuitive Surgical Inc., Sunnyvale, CA, USA) was docked at 45-degree in a counterclockwise fashion from the patient craniocaudal axis. Upon entering the thoracic cavity, the adhesions over the previous site of sympathectomy were released. Subsequently, we used a monopolar scissor, a Potts scissor, and a curved bipolar dissector to identify the scarred proximal and distal ends of the sympathetic trunk and the affected intercostal nerves (Figure 1). Upon identification of the 

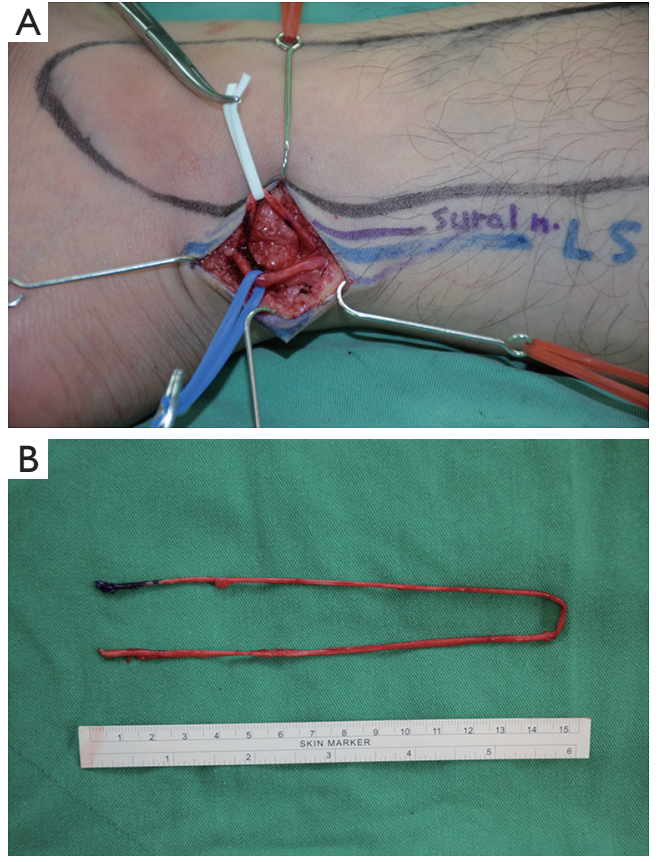

Figure 2 (A) The sural nerve was harvested from a small incision performed in the left lateral malleolus, behind the small saphenous vein; (B) harvesting and preparation of a $30-\mathrm{cm}$ sural nerve graft.

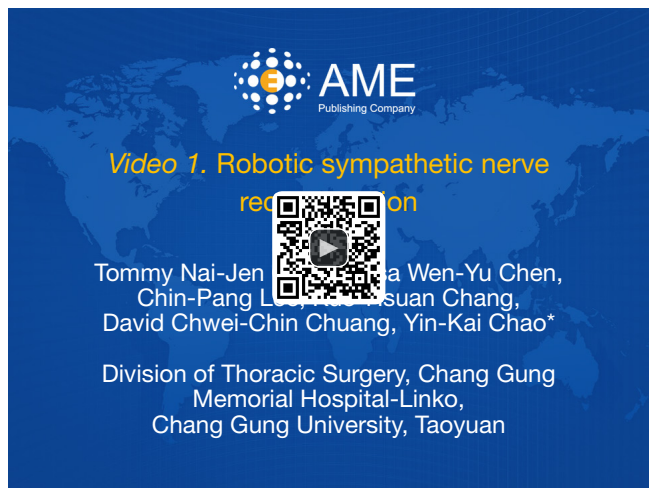

Figure 3 Robotic sympathetic nerve reconstruction (5). We initially identified the healthy stumps of (I) the proximal and distal sympathetic trunk and (II) the second-to-fourth intercostal nerves. We subsequently harvested the sural nerve-which was used to bridge the defect with the aid of a da $\mathrm{Vinci}^{\circledR}$ robotic system (black diamonds) and 9-0 nylon sutures. The sympathetic trunk defect was reconstructed and the second-to-fourth intercostal nerves were coapted back in an end-to-side fashion.

Available online: http://www.asvide.com/watch/33091 healthy stump, plastic surgeons started nerve harvesting. A retro-fibular incision was performed $3-5 \mathrm{~cm}$ cranially to the left ankle level to allow exploration of the sural nerve. The small saphenous vein and the sural nerve were marked with blue and white vessel loops, respectively. The dissection was performed in a proximal direction and a series of small stepwise incisions were performed to harvest the sural nerve (Figure 2A). Following its unequivocal identification (between the posterior tibial nerve and the common peroneal nerve, closer to the common peroneal nerve), the most proximal incision was performed on the popliteal crease. Upon cutting of the nerve, the wound was closed from the knee to the ankle after the bandage to avoid the formation of acuminated hematomas. In general, the harvested sural nerve was $25-30 \mathrm{~cm}$ in length and was used to repair the defect bilaterally (Figure 2B). The sural nerve was prepared on a side table and all of the tissue besides the epineurium were removed before its delivery to the chest. The proximal and distal sympathetic trunk stumps were cut at appropriate sites, with the nerve stumps required to be soft, pliable, fresh bleeding, and mushrooming over the fascicles. Coaptation of the sympathetic trunk and the ruptured intercostal nerves was performed using two "black diamond" micro-forceps and 8-0 or 9-0 nylon sutures (Figure 3). Using an inter-positional sural nerve graft, we initially performed a proximal coaptation to bridge the proximal sympathetic trunk (Figure $4 A$ ). We subsequently trimmed the graft to a length that allowed achieving a direct, tension-free suturing to the distal end of the sympathetic trunk (Figure 4B). We generally used five or six stitches for each coaptation. Subsequently, intercostal nerves were coapted back to the sural nerve graft either in an end-to-side (Figure $5 A$ ) or in a side-to-side (Figure 5B) fashion using three or four stitches. When the procedure was completed, hemostasis was checked carefully and a chest tube was placed through the assistant port. The same surgical approach was applied contralaterally. After surgery, patients were maintained in an intensive care unit (ICU) for overnight monitoring and subsequently transferred to a hospital ward on the next morning. In order to avoid nerve injuries, chest tubes-which were not connected to a low-pressure suction device-were maintained in their position for the minimum possible time. Upon removal of all drainage tubes, patients were 

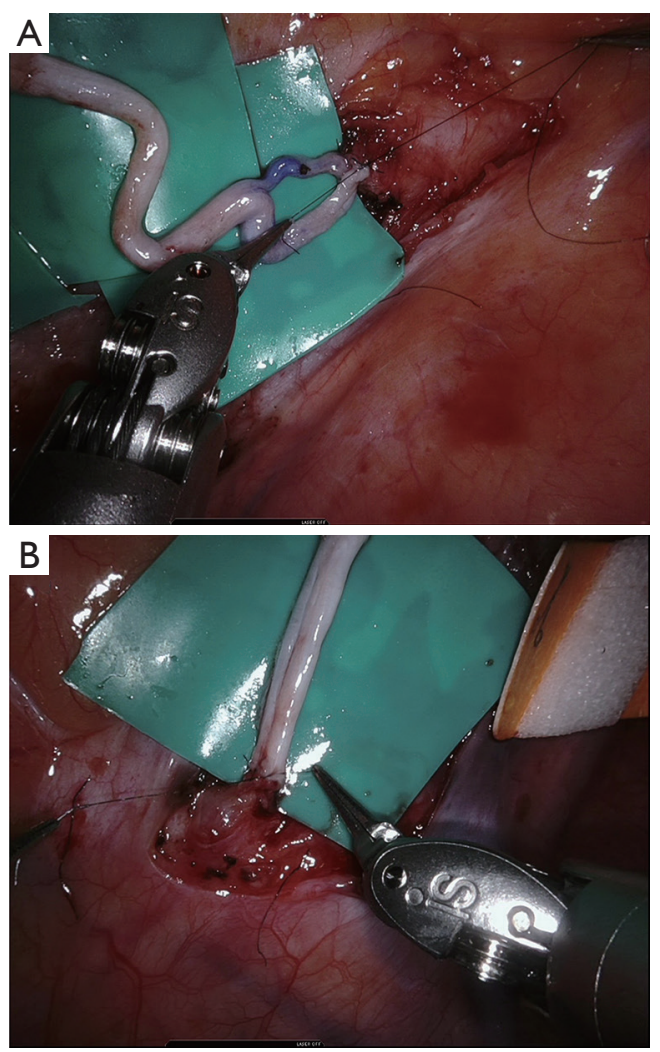

Figure 4 Proximal (A) and distal (B) end-to-end coaptation. We used 9-0 nylon sutures to bridge the sympathetic trunk stumps with the inter-positional sural nerve graft.

discharged on the third or fourth post-operative day.

\section{Postoperative follow-up}

Using a questionnaire focusing on the effects of surgery (Figure 6), patients were followed up every 6 months by phone interviews. Subjective symptoms were rated on a 10-point Likert scale (0: no symptoms; 10 : extremely severe symptoms).

\section{Results}

We identified seven consecutive patients who underwent robotic SNR between January 2017 and May 2019. The general characteristics of the patients are shown in Table 1. The study sample consisted of five men and two women (median age: 41 years). Primary hyperhidrosis affected the face in one case and the palms in six patients. ETS was initially performed at the T2-T3 level in four
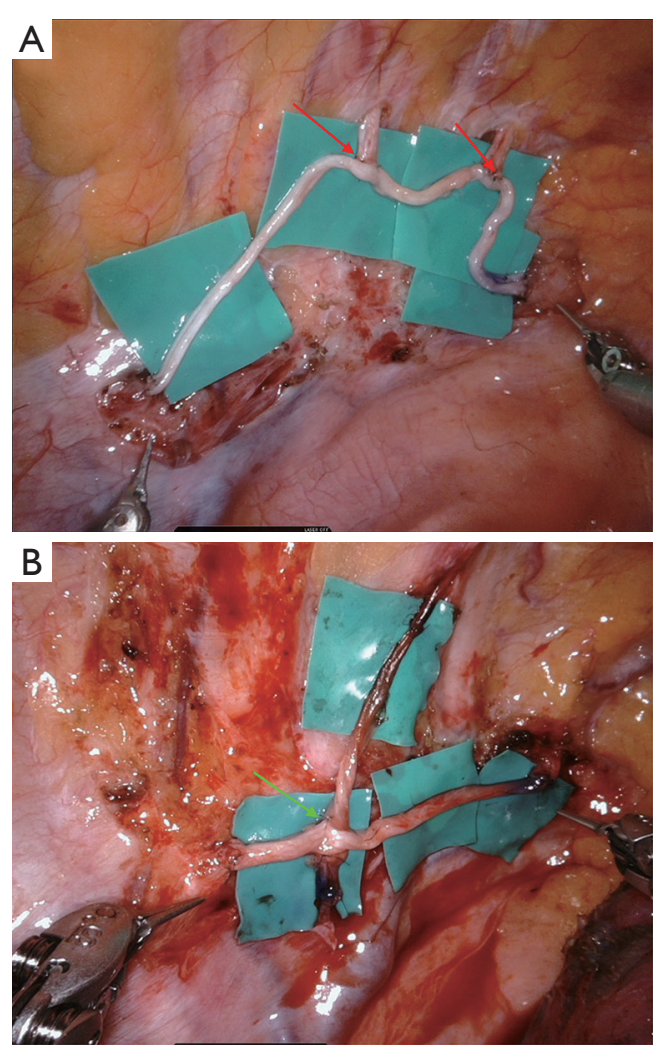

Figure 5 The ruptured intercostal nerves were coapted back to the sural nerve graft either in an end-to-side (A) or in a side-to-side (B) direction.

patients and at the T2-T4 levels in two patients. The remaining case received $\mathrm{T} 2-\mathrm{T} 3$ sympathectomy on the left side and T2-T4 sympathectomy on the right side. The median time from the ETS to SNR was 20 years. One case (patient \#5) received a second ETS with T5-T6 clipping for the treatment of CS. However, treatment results were unsatisfactory and clips were removed after 2 months.

The intraoperative and post-operative results of the seven cases are summarized in Table 2. The proximal and distal stumps of the transected sympathetic nerve were clearly identifiable in all patients. Reconstruction was performed with the sural nerve in all cases (median nerve graft length: $8 \mathrm{~cm}$ ). All robotic surgery procedures were successfully accomplished (median operating time: $10.5 \mathrm{~h}$ ), and neither conversion to open surgery nor the creation of additional ports were required. There was no operative mortality, and the median length of post-operative hospital stay was 4 days. One patient developed a post-operative pneumothorax upon chest tube removal—which was treated 


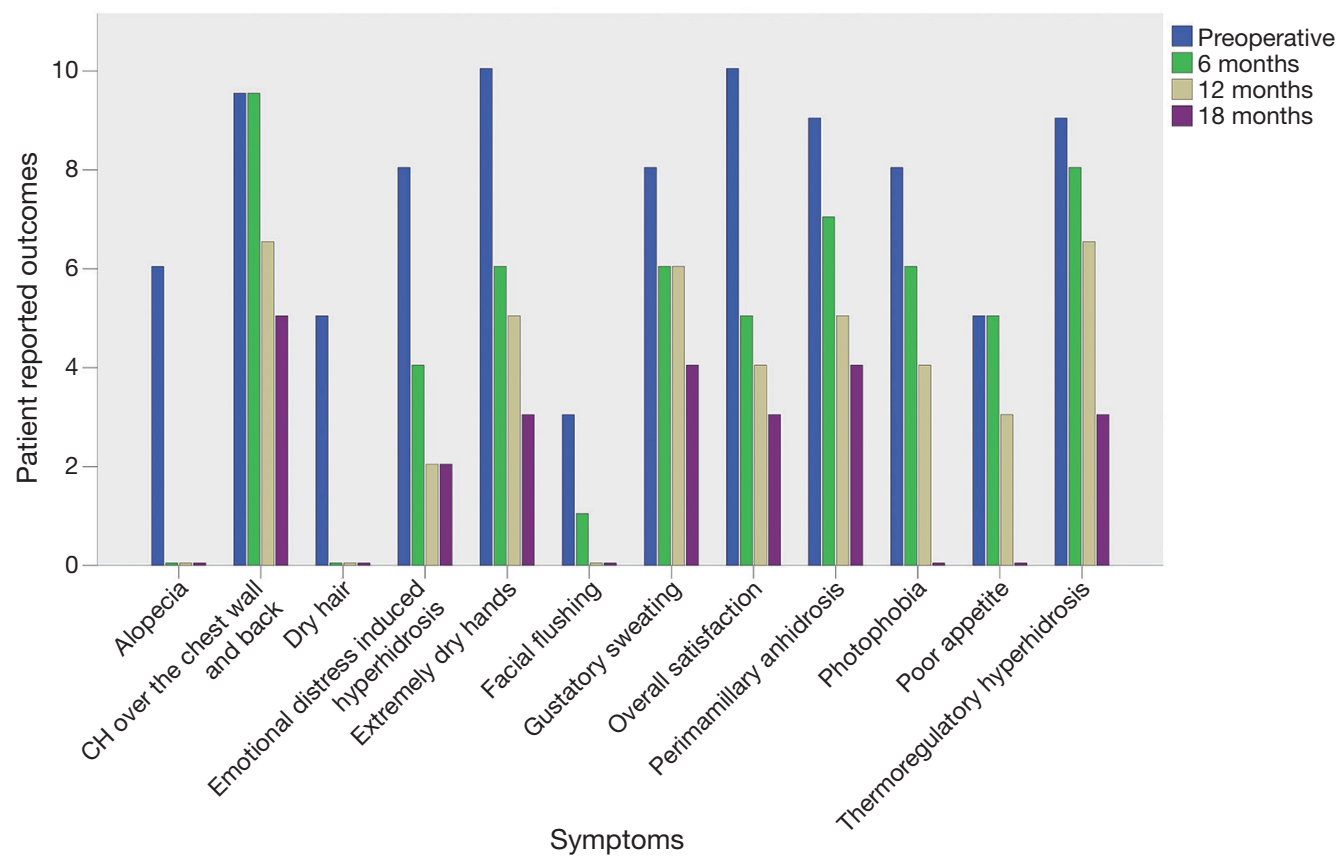

Figure 6 Patient-reported outcomes in a case who received SNR more than 6 months before. CH, compensatory hyperhidrosis; SNR, sympathetic nerve reconstruction.

Table 1 General characteristics of the seven study patients

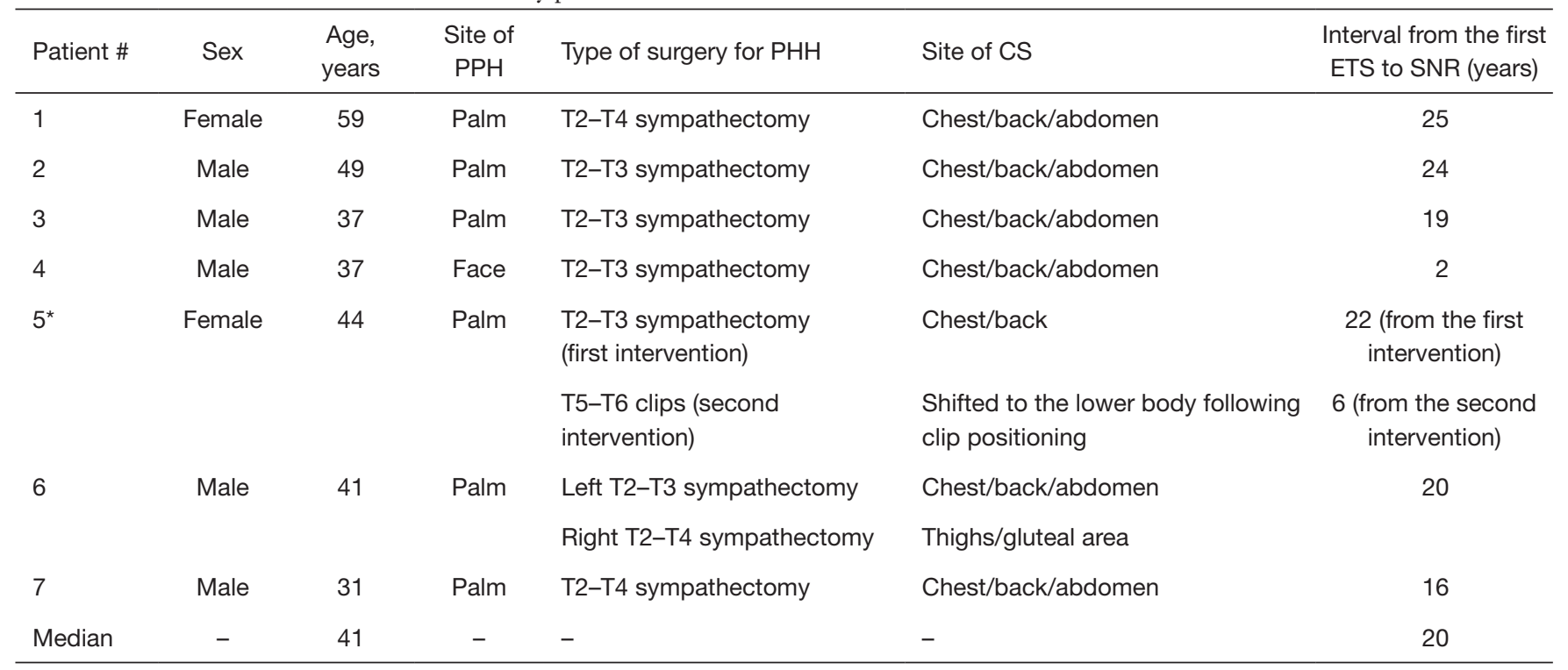

${ }^{*}$, the patient underwent two distinct ETS operations. PPH, primary hyperhidrosis; CS, compensatory sweating; ETS, endoscopic thoracic sympathectomy; SNR, sympathetic nerve reconstruction. 
Table 2 Procedural details and surgical outcomes

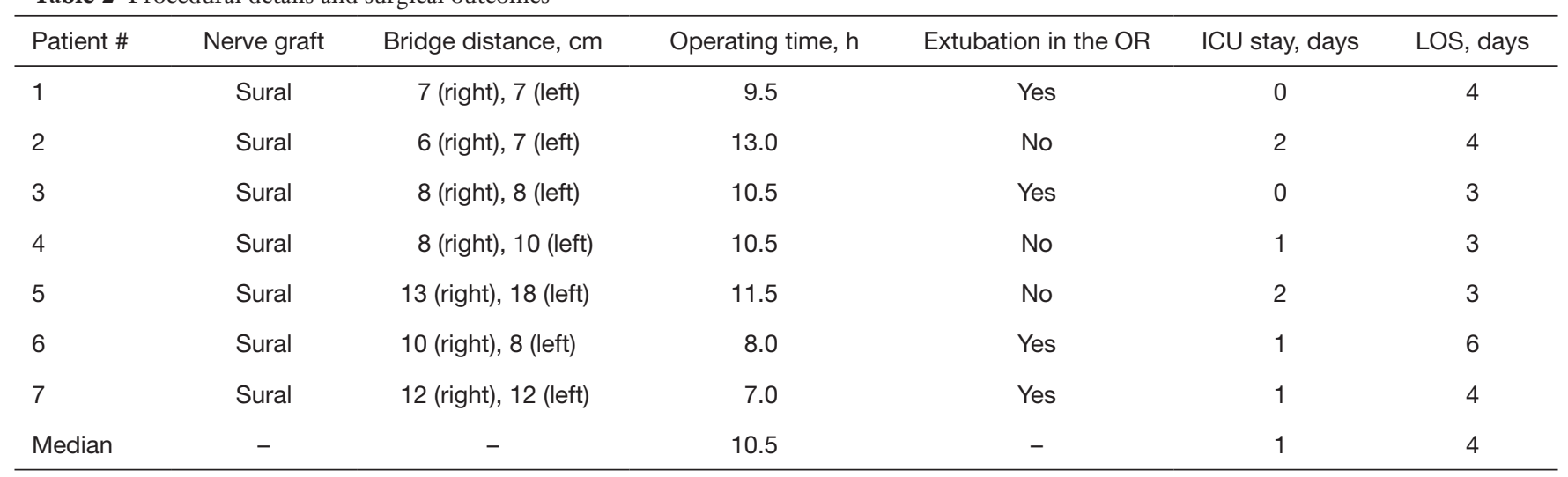

OR, operating room; ICU, intensive care unit; LOS, length of stay.

Table 3 Published reports on sympathetic nerve reconstruction using a minimally invasive surgical approach

\begin{tabular}{|c|c|c|c|c|}
\hline Authors, year of publication & Number of patients & Surgical approach & Graft type & Nerve coaptation \\
\hline Wong et al., 2012 (4) & 1 & Thoracoscopy & Intercostal nerve & Fibrin glue \\
\hline Park et al., 2014 (9) & 2 & Thoracoscopy & Vein graft & Fibrin glue \\
\hline Connery et al., 2016 (10) & 3 & Robot-assisted surgery & Sural nerve $(n=1)$, intercostal nerve $(n=2)$ & Suture \\
\hline Current study & 7 & Robot-assisted surgery & Sural nerve & Suture \\
\hline
\end{tabular}

conservatively.

Because of the short follow-up period, data on symptom improvements were available for one patient only (case \#1) who received SNR more than 6 months before. Figure 6 shows a $70 \%$ improvement compared with the pre-operative period in terms of ETS-induced symptoms. The most remarkable improvements were observed in the following areas: extremely dry hands (score change: from 10 to 3 ), photophobia (score change: from 7 to 0 ), thermoregulation (score change: from 9 to 3), emotional health (score change: from 8 to 2), and anhidrosis above the nipple level (score change: from 9 to 4$)$.

\section{Discussion}

Previous experimental studies have focused on the feasibility of SNR as a surgical rescue treatment for post-operative CS (6). In 1998, Telaranta (7) performed a unilateral SNR in a patient who developed post-sympathectomy
CS accompanied by severe exercise intolerance. Their technique consisted of an open thoracotomy, with the sural nerve being used as an interposition graft. Several studies have described a thoracoscopic approach to SNR-using either intercostal or sural nerve or vein grafting $(3,4,8,9)$. However, published data (Table 3) have been inconsistent and poorly reproducible both because of technical (i.e., instrument rigidity, two-dimensional vision) and anatomical (i.e., narrow surgical space) reasons. Delicate nerve coaptation during thoracoscopy remains technically challenging and generally requires the use of fibrin glues in the absence of suturing $(3,4,8,9)$. In this scenario, robotassisted microsurgical reconstruction of the sympathetic trunk represents a potential breakthrough in the field of SNR. However, there has been only one report describing this approach in a limited case series consisting of three patients (10). Although the results were promising, the limited sample size did not allow to draw firm conclusions on the clinical utility of this technique. To our knowledge, 
this is the largest series to date describing the feasibility and clinical outcomes of robotic SNR. Notably, the sympathetic nerve defect was uniformly reconstructed using the sural nerve in all of the seven study participants.

We believe that the approach described in our current study can offer several advantages over the traditional thoracoscopic technique. First, the three-dimensional, self-controlled $10 \times$ magnified view provided by the robotic camera allows a better visualization, permitting an easier identification of the healthy proximal stump of the sympathetic trunk. Second, robot-assisted surgery facilitates a meticulous suturing with a $8-0$ nylon even in a limited anatomical space. This is achieved both by tremor filtering and through the use of articulated micro-instrumentation designed to have seven degrees of freedom. All of these features are expected to increase the rates of successful SNR.

Although the robotic approach described herein should be considered as minimally invasive, we were unable to extubate three patients within the operating room. This in turn required the implementation of short-term (6-8 h) post-operative mechanical ventilation. This phenomenon can be ascribed not only to a prolonged anesthesia time but also to the inability of the sympathetic nervous system to respond properly following ETS. Because post-ganglionic sympathetic fibers originating from neurons residing in the cervico-thoracic ganglia are involved in the innervation of thoracic organs (including the heart and lungs), ETS may at least in part impair cardio-respiratory function in certain patients (11).

Despite the promising results reported in our case series, some limitations of our report merit comment. Specifically, our data should be considered as preliminary and are limited by the single-center nature of the study. Owing to the small sample size and the limited follow-up period, our findings need independent replication before drawing more definitive conclusions on the usefulness of the proposed robotic approach. Larger studies with higher statistical power are also warranted to investigate the success rates of robotic SNR in the reversal of CS.

\section{Conclusions}

Our case series demonstrates the safety and clinical feasibility of microsurgical robot-assisted sural nerve grafting for achieving SNR in patients with post-operative CS.

\section{Acknowledgments}

Funding: This study was financially supported by a grant (CMRPG3H0952) from the Chang Gung Memorial Hospital.

\section{Footnote}

Conflicts of Interest: The authors have no conflicts of interest to declare.

Ethical Statement: The authors are accountable for all aspects of the work in ensuring that questions related to the accuracy or integrity of any part of the work are appropriately investigated and resolved. The Institutional Review Board of the Chang Gung Memorial Hospital approved the study protocol (CGMH-IRB 201901142B0). All participants gave their written informed consent.

\section{References}

1. Bryant AS, Cerfolio RJ. Satisfaction and compensatory hyperhidrosis rates 5 years and longer after video-assisted thoracoscopic sympathotomy for hyperhidrosis. J Thorac Cardiovasc Surg 2014;147:1160-3.e1.

2. Stefaniak T, Cwigon $M$, Łaski D. In the search for the treatment of compensatory sweating. ScientificWorldJournal 2012;2012:134547.

3. Haam SJ, Park SY, Paik HC, et al. Sympathetic nerve reconstruction for compensatory hyperhidrosis after sympathetic surgery for primary hyperhidrosis. J Korean Med Sci 2010;25:597-601.

4. Wong RH, Ng CS, Wong JK, et al. Needlescopic video-assisted thoracic surgery for reversal of thoracic sympathectomy. Interact Cardiovasc Thorac Surg 2012;14:350-2.

5. Chang TN, Chen LW, Lee CP, et al. Robotic sympathetic nerve reconstruction. Asvide 2020;7:051. Available online: http://www.asvide.com/watch/33091

6. Latif MJ, Afthinos JN, Connery CP, et al. Robotic intercostal nerve graft for reversal of thoracic sympathectomy: a large animal feasibility model. Int J Med Robot 2008;4:258-62.

7. Telaranta T. Secondary sympathetic chain reconstruction 
after endoscopic thoracic sympathicotomy. Eur J Surg Suppl 1998;164:17-8.

8. Rantanen T, Telaranta T. Long-Term effect of endoscopic sympathetic nerve reconstruction for side effects after endoscopic sympathectomy. Thorac Cardiovasc Surg 2017;65:484-90.

9. Park HS, Hensman C, Leong J. Thoracic sympathetic nerve reconstruction for compensatory hyperhidrosis: the
Melbourne technique. Ann Transl Med 2014;2:45.

10. Connery CP. Reconstruction of the sympathetic chain. Thorac Surg Clin 2016;26:427-34.

11. Fiorelli A, D'Aponte A, Canonico R, et al. T2-T3 sympathectomy versus sympathicotomy for essential palmar hyperhidrosis: comparison of effects on cardio-respiratory function. Eur J Cardiothorac Surg 2012;42:454-61.
Cite this article as: Chang TN, Chen LW, Lee CP, Chang $\mathrm{KH}$, Chuang DC, Chao YK. Microsurgical robotic suturing of sural nerve graft for sympathetic nerve reconstruction: a technical feasibility study. J Thorac Dis 2020;12(2):97-104. doi: 10.21037/jtd.2019.08.52 